\title{
Tratamiento informativo de los mapuches en el diario El Mercurio. Un análisis de contenido desde la teoría del framing ${ }^{1}$
}

Recibido: 5 de marzo de 2019 Aceptado: 30 de mayo de 2019

Publicado: 24 de junio de 2019

\author{
Carlos del Valle-Rojas \\ carlos.delvalle@ufrontera.cl \\ Universidad de La Frontera (Chile) \\ María José Ufarte Ruiz \\ mariajose.ufarte@uclm.es \\ Universidad de Castilla-La Mancha (España) \\ Francisco José Murcia Verdú \\ fjmurcia001@ikasle.ehu.eus \\ Universidad del País Vasco (España) \\ Francisca Silva-Layera \\ francisca.silva@ufrontera.cl \\ Universidad de La Frontera (Chile)
}

Resumen: Este trabajo analiza el tratamiento informativo del pueblo mapuche en el diario El Mercurio durante dos períodos. Primero, entre los años 1950 y 1973 (siglo XX); y segundo, durante los años 2010, 2013 y 2014, (siglo XXI). Para ello se ha seleccionado un corpus de 340 textos periodísticos (unidades de análisis), de los que 147 corresponden al siglo XX (1950-1970) y 193 al siglo XXI (2010, 2013-2014). El análisis muestra que la mayoría de los encuadres están vinculados a los conflictos, los procesamientos penales y los ataques incendiarios; de modo que El Mercurio prioriza connotaciones y sucesos negativos para informar sobre los indígenas mapuches.

Palabras clave: Grupo étnico, prensa de información diaria, análisis de contenido, atribución de frecuencia, Chile ${ }^{2}$.

1 Este trabajo es financiado por el Proyecto CONICYT-Chile PIA/ANILLOS SOC180045; y por el Proyecto CONICYT-Chile FONDECYT 1150666..

2 Tesauro de la Unesco. 
Abstract: This paper analyzes the information treatment of the Mapuche people in the newspaper El Mercurio during two periods. First, between 1950 and 1973 (twentieth century); and second, during the years 2010, 2013 and 2014, (21st century). To this end, a corpus of 340 journalistic texts has been selected, of which 147 correspond to the 20th century (1950-1970) and 193, to the 21st century (2010, 2013-2014). The analysis shows that most of the frames are linked to conflicts, criminal prosecutions and arson attacks; so that El Mercurio prioritizes connotations and negative events to inform about the Mapuche indigenous people.

Key words: Ethnic Group, Newspaper Press, Content Analysis, Allocation of Frequencies, Chile.

\section{Introducción}

El Mercurio, periódico de circulación diaria en Chile, asume un rol político que forma parte, a su vez, de una compleja industria cultural, cuyo propósito es producir y reproducir al indígena mapuche como enemigo íntimo del proceso de "civilización" y "desarrollo" que el Estado nacional viene implementando durante los siglos XIX, XX y XXI como una estrategia institucionalizada y sistemática (Del Valle, 2018, 2019).

Sin embargo, aún existen escasos estudios que analicen en profundidad el tratamiento informativo de la comunidad mapuche en los medios de comunicación. Las investigaciones realizadas hasta el momento se centran, básicamente, en aspectos históricos (Bengoa, 2000; Del Valle, 2016), interculturales (Del Valle, 2007; Maldonado \& Del Valle, 2016), de resistencia y reestructuración (Boccara, 1999; Del Valle, 2003), asociados con la propiedad de la tierra (Almonacid, 2009) y al discurso periodístico (Del Valle, 2004a), público (Carrasco, 2002, Maldonado, 2014) y jurídico-judicial (Del Valle, 2002). También se han analizado sus derechos y desafíos para la sustentabilidad democrática (Toledo, 2005; Del Valle, Davinson \& Maldonado, 2015); la presencia de estereotipos (Saiz, Merino \& Quilaqueo, 2009; Saiz, Rapimán \& Mladinic, 2008); y las representaciones sociales en torno al conflicto (Denegri, Chávez, Silva \& Del Valle, 2015). Incluso, existen investigaciones que abordan el desplazamiento del conflicto mapuche al espacio virtual (Maldonado, 2014; Maldonado \& Del Valle, 2013) y la conformación de la agenda pública chilena acerca del pueblo mapuche y del conflicto que este mantiene con el Estado chileno (Del Valle, 2004a; Salinas \& Stange, 2009; Del Valle, Mellado, Salinas \& González, 2011; Del Valle, 2015).

En este sentido, y en términos más específicos, encontramos el estudio de Labrín (2011), que analiza de manera genérica la cobertura y tratamiento de conflictos indígenas en 2010 y 2011, y el proyecto financiado por la Comisión Nacional de Investigación Científica y Tecnológica (CONICYT) PLU número 140014 (Hudson, Del Valle, Dussaillant \& Browne, 2017-2018), que aborda el diseño de un indicador de pluralismo aplicado a la tematización de la agenda de la prensa escrita y a la validación de las fuentes, tomando como caso de estudio la cobertura del tema mapuche en la prensa nacional y en la prensa regional. 
Se trata de una amplia variedad temática y de encuadres que debe completarse desde otras perspectivas. De ahí, que el objetivo de la presente investigación sea analizar el tratamiento informativo de los mapuches en el diario El Mercurio durante los años 1950-1973 (siglo XX) y 2010, 2013 y 2014 (siglo XXI). Además, este estudio pretende corroborar las siguientes hipótesis:

- H1: Los textos periodísticos que publica el diario El Mercurio sobre este colectivo son de carácter despectivo y discriminatorio.

- H2: Los conflictos, los procesamientos penales y los ataques incendiarios son los principales encuadres noticiosos que se derivan del tema mapuche en El Mercurio, en sintonía a lo que demuestran otras investigaciones previas, como la de (Amolef, 2004).

- H3: El tratamiento informativo de este colectivo se suele realizar a partir de una variedad de dimensiones temáticas, lo que otorga una mayor contextualización informativa.

En las siguientes líneas se recoge la literatura científica para entender el contexto, se presenta la metodología utilizada y se explican y discuten los resultados obtenidos en la presente investigación.

\section{Marco referencial. La teoría del framing y el colectivo mapuche}

Los medios de comunicación tienen la capacidad de organizar, elaborar y tratar la información a través de diferentes enfoques o puntos de vista sobre un mismo tema (McCombs \& Shaw, 1972; Scheufele, 2004, 2009; Igartua \& Humanes, 2004; Muñiz, 2015). Este es el planteamiento básico que establece la teoría del encuadre o framing, según la terminología anglosajona (Gitlin, 1980; Entman, 1993; Reese, 2001; Tankard, 2001; D’Angelo, 2002; De Vreese, 2003; Scheufele, 2004; Bryant \& Miron, 2004; Weaver, 2007; López \& Vicente, 2008; Scheufele, 1999; López-Rabadán, 2010; Koziner, 2013, Marín \& Zamora, 2014, Oller, 2014), que propone el análisis de encuadres noticiosos (news frames) empleados por los periodistas para enfocar la realidad social en los textos publicados.

El concepto de framing ha sido objeto de un progresivo proceso de definición teórica, pasando por diferentes fases de evolución de conceptualización y operacionalización desde la década de 1970 hasta la actualidad (López-Rabadán, 2010). No obstante, en las dos últimas décadas se ha podido percibir un constante aumento de los estudios que recurren a los encuadres como una herramienta conceptual básica (Vicente \& López, 2009), lo que pone de relieve los avances en la comprensión del concepto y, a su vez, las carencias todavía existentes. De este modo, el investigador que se adentra en el estudio de esta materia se enfrenta a una gran disparidad de definiciones, que en ocasiones resultan contradictorias (Entman, 1993; McCombs, 2006; Van Gorp, 2007).

Para Entman (1993), el proceso de encuadrar o framing implica la selección de algunos aspectos de la realidad, a los que les otorga un mayor énfasis o importancia, en detrimento de otros, de manera que se puede influir en la audiencia (Guo, Hong \& McCombs, 2012; 
Koziner \& Aruguete, 2016). De ahí que Sádaba (2001) considere que esta teoría rechaza los principios de objetividad, ya que cuando el periodista encuadra la realidad y cuenta lo que sucede, está aportando su punto de vista. El propósito objetivista (de ofrecer diferentes versiones sobre un tema para que el lector escoja) encierra, a juicio de la autora, una perspectiva de la verdad como si fuese objeto de elección.

Sin embargo, hay que tener en cuenta que todo texto noticioso presenta omisiones, deliberadas o no, pues es imposible abordar un asunto desde todas sus perspectivas, utilizando todas las fuentes posibles y explicando el papel de todos los actores implicados directa e indirectamente en el problema (Ardèvol-Abreu, 2015).

En el ámbito periodístico, Maher (2001) y Muñiz (2015) consideran que el framing implica una relación entre los elementos existentes en el mensaje, mientras que Reese (2001) sostiene que alude a la manera en la que los eventos y asuntos son organizados y se les da sentido por parte de los medios de comunicación, los profesionales de los medios y sus audiencias. Es, en cualquier caso, una estructura que guía nuestro conocimiento sobre el origen de un problema determinado (Thibodeau, 2017), y ofrece sugerencias para poder evaluar las soluciones posibles (Muñiz, Igartua, Otero y Sánchez, 2008).

\section{Metodología}

Para el análisis de textos, hemos seleccionado el periódico El Mercurio, que es el más representativo de Chile, por historia, tirada y audiencia, durante los dos periodos estudiados (siglo XX: 1950-1973, y siglo XXI: 2010, 2013 y 2014), según la Asociación de Agencia de Medios (AAM, 2018). En efecto, El Mercurio fue fundado el 12 de septiembre de 1827 como El Mercurio de Valparaíso y desde el 1 de junio de 1900 recibe la denominación de El Mercurio de Santiago.

La localización y elección de las unidades de análisis se llevó a cabo como parte del proyecto de investigación del que forma parte el presente trabajo, denominado "La construcción del imaginario social de la justicia en los relatos periodísticos publicados por el diario El Mercurio de Chile, entre 1850 y 2014, en el contexto del conflicto Estado-nación y pueblo mapuche: Continuidades y cambios", financiado por el Fondo Nacional de Desarrollo Científico y Tecnológico-FONDECYT, número 1150666. Para la selección del corpus, se consideró la comparación de dos períodos de El Mercurio correspondientes a los siglos XX y XXI, específicamente aquellos textos periodísticos de información, interpretación y opinión que tengan alguna referencia directa o indirecta a las relaciones entre el Estado nacional chileno y el pueblo mapuche. En este proceso se seleccionó un total de 340 publicaciones, que constituyen el corpus de análisis. 
Tabla 1: Selección del corpus.

\begin{tabular}{|l|l|l|l|l|}
\hline \multirow{2}{*}{} & \multicolumn{3}{|c|}{ Criterios generales } & \multicolumn{2}{c|}{ Criterios específicos } \\
\cline { 2 - 5 } Criterios/ & $\begin{array}{l}\text { Textos periodís- } \\
\text { ticos del género } \\
\text { informativo, } \\
\text { interpretativo y } \\
\text { de opinión }\end{array}$ & $\begin{array}{l}\text { Textos periodísticos de } \\
\text { referencia a las relaciones } \\
\text { entre el Estado nacional } \\
\text { chileno y el pueblo } \\
\text { mapuche }\end{array}$ & $\begin{array}{l}\text { Textos } \\
\text { periodísticos } \\
\text { correspondientes } \\
\text { al siglo XX (1950- } \\
1970)\end{array}$ & $\begin{array}{l}\text { Textos periodísticos } \\
\text { correspondientes al } \\
\text { siglo XXI (2010, } \\
2013-2014)\end{array}$ \\
\hline 340 textos & & & 147 & 193 \\
\hline
\end{tabular}

Fuente: Elaboración propia.

Una vez definida la muestra, realizamos un proceso de codificación de los datos a partir de un diseño preliminar de categorías (Bardin, 2002; Sánchez Aranda, 2005), que nos permitió delimitar las unidades de análisis en unidades de contexto (texto periodístico) y de registro (párrafos, oraciones o ideas temáticas que presentan los textos publicados). Para ello, se generó una plantilla de codificación inicial que, tras un estudio piloto, quedó conformada en 8 categorías y 70 subcategorías.

Como unidades de contexto se incluyeron las siguientes:

- Fecha: desde 1950 hasta 1973; 2010, 2013, 2014.

- Sección: "Nacional", "Opinión”.

- Género periodístico: breve periodístico, noticia, crónica, entrevista, reportaje, cartas al director, artículo de opinión.

- Morfología del texto: breve, extenso.

Como unidades de registro se contemplaron las que a continuación se detallan:

- Valoración: positiva, negativa, neutra.

- Tipo de lenguaje: afectivo, despectivo, objetivo.

- Dimensión temática: acciones reivindicativas y protestas, ataques incendiarios, atribución de responsabilidad, conflicto, crisis, cultura y educación, economía, empleo, enfrentamientos, interés humano, judicatura, legislación indígena, moral, presencia policial, procesamientos penales, religión, resolución de problemas y conflictos (agricultura, modificación de ley, salud, terrenos), sociales.

- Términos con los que se alude al pueblo mapuche: araucano/s, indígena/s, indio/s y mapuche/s.

Estos ítems medían la frecuencia con la que se publicaba un mismo tema en el diario El Mercurio y estaban dedicadas al estudio de los encuadres noticiosos (tabla 2). Nuestra ficha de análisis contiene, por tanto, las cuatro dimensiones de los atributos o frames propuestos por Ghanem (2009):

- El tópico de una noticia: qué debe ser incluido en el frame.

- La presentación: que incluye la morfología del texto y ubicación, que en nuestro caso es la sección periodística. 
- Los atributos cognitivos: entendidos como aquellos detalles que son incluidos en el frame.

- Los atributos afectivos: como el tono (valoración y tipo de lenguaje).

También cuenta con los cinco encuadres propuestos por Semetko \& Valkenburg (2000):

- Conflicto.

- De interés humano, con el que se suele presentar un tratamiento emocional de un problema.

- De consecuencias económicas del hecho narrado.

- De moralidad.

- De atribución de responsabilidad del asunto o problema objeto de información.

Son, en definitiva, atributos descriptivos que integran el mensaje y caracterizan un objeto que define el tema o idea central (Aruguete, 2017).

Tabla 2: Selección del corpus.

\begin{tabular}{|c|c|c|}
\hline \multicolumn{3}{|c|}{ FICHA TÉCNICA DE ANÁLISIS } \\
\hline Categorías & Subcategorías & Observaciones \\
\hline \multirow{27}{*}{ Fecha } & 1950 & \\
\hline & 1951 & \\
\hline & 1952 & \\
\hline & 1953 & \\
\hline & 1954 & \\
\hline & 1955 & \\
\hline & 1956 & \\
\hline & 1957 & \\
\hline & 1958 & \\
\hline & 1959 & \\
\hline & 1960 & \\
\hline & 1961 & \\
\hline & 1962 & \\
\hline & 1963 & \\
\hline & 1964 & \\
\hline & 1965 & \\
\hline & 1966 & \\
\hline & 1967 & \\
\hline & 1968 & \\
\hline & 1969 & \\
\hline & 1970 & \\
\hline & 1971 & \\
\hline & 1972 & \\
\hline & 1973 & \\
\hline & 2010 & \\
\hline & 2013 & \\
\hline & 2014 & \\
\hline
\end{tabular}




\begin{tabular}{|c|c|c|}
\hline \multirow{2}{*}{ Sección } & Nacional & \\
\hline & Opinión & \\
\hline \multirow{7}{*}{$\begin{array}{l}\text { Género } \\
\text { periodístico }\end{array}$} & Breve periodístico & \\
\hline & Noticia & \\
\hline & Crónica & \\
\hline & Entrevista & \\
\hline & Reportaje & \\
\hline & Artículo de opinión & \\
\hline & Cartas al director & \\
\hline \multirow{2}{*}{$\begin{array}{l}\text { Morfología del } \\
\text { texto }\end{array}$} & Breve & \\
\hline & Extenso & \\
\hline \multirow{3}{*}{ Valoración } & Positiva & \\
\hline & Negativa & \\
\hline & Neutral & \\
\hline \multirow{3}{*}{ Tipo de lenguaje } & Afectivo & \\
\hline & Despectivo & \\
\hline & Objetivo & \\
\hline & Acciones reivindicativas y protestas & \\
\hline & Ataques incendiarios & \\
\hline & Atribución de responsabilidad & \\
\hline & Conflicto & \\
\hline & Crisis & \\
\hline & Cultural y educación & \\
\hline & Economía & \\
\hline & Empleo & \\
\hline & Enfrentamientos & \\
\hline & Interés humano & \\
\hline Dimensión & Judicatura & \\
\hline temática & Legislación indígena & \\
\hline & Moral & \\
\hline & Presencia policial & \\
\hline & Procesamientos penales & \\
\hline & Religión & \\
\hline & Resolución de problemas y conflictos & \\
\hline & \begin{tabular}{|l|l|} 
& Agricultura \\
\end{tabular} & \\
\hline & Modificación de ley & \\
\hline & Salud & \\
\hline & Terrenos & \\
\hline & Social & \\
\hline & Mapuche/s & \\
\hline Términos & Indio/s & \\
\hline utilizados & Indígena/s & \\
\hline & Araucano/s & \\
\hline
\end{tabular}

Fuente: Elaboración propia.

Los textos han sido tratados con la ayuda del software NVivo 12, un programa diseñado para el análisis de datos cualitativos que facilita la codificación y análisis de documentos de trabajo. Su utilización en el marco de la presente investigación nos ha permitido almacenar, 
organizar y obtener informes resumidos de los datos más significativos que emergen de nuestro análisis. Después de esta fase, hemos procedido a la extracción de las nubes de palabras y mapas ramificados para ver los conceptos más frecuentes en los materiales seleccionados. Este análisis previo (de doble dimensión, cuantitativa y cualitativa) hace mucho más fácil y viable el posterior análisis del discurso.

Para interpretar los resultados y poder realizar las inferencias pertinentes a lo largo de todo el estudio, empleamos el análisis de contenido (Berelson, 1952; Weber, 1990; Van Dijk, 2000; Krippendorff, 2002; Bardin, 2002; Sánchez Aranda, 2005), debido a que desde el punto de vista metodológico, el framing requiere del empleo de otras técnicas para analizar los datos (Aruguete, 2017). Andréu Abela, citado por Krippendorff (2002), explica que el análisis de contenido "se basa en la lectura (textual o visual) como instrumento de recogida de información, lectura que [...] debe ser sistemática, objetiva, replicable, y válida". Por ese motivo, es necesario hallar en el texto en cuestión unas determinadas variables, ordenadas y clasificadas previamente por categorías, que han de proporcionar de forma sistemática datos cuantificables, validables, que pueden cruzarse, contrastar y comparar para poder llegar de este modo a conclusiones válidas (Krippendorff, 2002). No obstante, debe ser la combinación del análisis cuantitativo y el análisis interpretativo la que palíe o minimice las limitaciones del primero, de acuerdo con lo que propone Sáez (1999).

\section{Análisis de los resultados}

\subsection{Análisis de la cobertura del colectivo mapuche}

Del total del corpus analizado $(\mathrm{N}=340), 147$ textos corresponden al periodo comprendido entre 1950 y 1973, 16 informaciones son publicadas en 2010, mientras que las 177 restantes corresponden a 2013 y 2014 (tabla 3).

Tabla 3: Número de textos publicados, por años y género periodístico.

\begin{tabular}{|c|c|c|c|c|}
\hline Años & $\mathrm{N}^{0}$ de textos & \multicolumn{3}{|l|}{ Géneros } \\
\hline & & & $\mathrm{N}^{\mathrm{o}}$ & $\%$ \\
\hline \multirow{3}{*}{$1950-1973$} & \multirow{3}{*}{147} & Breves periodísticos & 34 & $23.30 \%$ \\
\hline & & Noticias & 57 & $39.0 \%$ \\
\hline & & Crónicas & 56 & $37.70 \%$ \\
\hline \multicolumn{3}{|c|}{ Total primer periodo } & 147 & $100 \%$ \\
\hline & & & $\mathrm{N}^{\mathrm{o}}$ & $\%$ \\
\hline \multirow{5}{*}{2010} & \multirow{5}{*}{16} & Noticias & 3 & $18.75 \%$ \\
\hline & & Crónicas & 6 & $37.50 \%$ \\
\hline & & Reportajes & 2 & $12.50 \%$ \\
\hline & & Cartas al director & 2 & $12.50 \%$ \\
\hline & & Artículos de opinión & 3 & $18.75 \%$ \\
\hline \multicolumn{3}{|c|}{ Total segundo periodo } & 16 & $100 \%$ \\
\hline
\end{tabular}




\begin{tabular}{|l|l|l|l|l|}
\hline \multicolumn{2}{|l|}{} & \multicolumn{1}{|c|}{$\mathrm{N}^{\mathrm{o}}$} & \multicolumn{1}{c|}{$\%$} \\
\hline \multirow{3}{*}{$2013-2014$} & \multirow{3}{*}{177} & Noticias & 107 & $60.45 \%$ \\
\cline { 3 - 5 } & Crónicas & 59 & $33.34 \%$ \\
\cline { 3 - 5 } & Entrevistas & 1 & $0.56 \%$ \\
\cline { 3 - 5 } & Reportajes & 10 & $5.65 \%$ \\
\hline Total tercer periodo & & 177 & $100 \%$ \\
\hline Total tres periodos & & 340 & $100 \%$ \\
\hline
\end{tabular}

Fuente: Elaboración propia.

Adentrándonos en cada periodo de estudio, es posible comprobar que las noticias tienen una mayor presencia sobre el resto de géneros periodísticos. En total, se han publicado 167 noticias (49.12\%), seguidas de 121 crónicas $(35.59 \%)$ y 34 breves periodísticos $(10.00 \%)$. En menor medida, encontramos reportajes $(3.53 \%)$, artículos de opinión $(0.88 \%)$, cartas al director $(0.59 \%)$ y entrevistas $(0.29 \%)$ (tabla 4$)$.

Tabla 4: Número de textos publicados en el corpus general de estudio..

\begin{tabular}{|l|r|r|}
\hline \multicolumn{1}{|c|}{ Géneros periodísticos } & \multicolumn{1}{c|}{$\mathbf{N}^{\mathbf{0}}$ de textos publicados } & \% Total \\
\hline Noticias & 167 & $49.12 \%$ \\
\hline Crónicas & 121 & $35.59 \%$ \\
\hline Breves periodísticos & 34 & $10.00 \%$ \\
\hline Reportajes & 12 & $3.53 \%$ \\
\hline Artículos de opinión & 3 & $0.88 \%$ \\
\hline Cartas al director & 2 & $0.59 \%$ \\
\hline Entrevistas & 1 & $0.29 \%$ \\
\hline Total: 340 & & $100 \%$ \\
\hline
\end{tabular}

Fuente: Elaboración propia.

El análisis también demuestra cómo la mayoría de los textos sobre los mapuches (335) ha sido emplazada dentro de la sección de "Nacional" (98.53\%), seguido a distancia por el porcentaje de publicaciones incorporadas en la sección de "Opinión”, que queda reducida a un porcentaje muy bajo (1.47\%). Estas cifras indican que El Mercurio utiliza de forma predominante la primera sección reseñada para presentar la información sobre el colectivo indígena.

En cuanto a la morfología, hay 202 textos extensos (59.41\%), que se localizan sobre todo en los últimos periodos estudiados, frente a 138 publicaciones breves $(40.59 \%)$, que predominan en la primera etapa analizada. De hecho, los breves periodísticos que forman parte del corpus de estudio pertenecen a esta época, donde abundan las informaciones construidas con frases cortas y de modo directo.

En cuanto a la forma de referirse al colectivo, existen diferencias significativas. Entre 1950 y 1973, predomina el término "Indígena/s" (50.74\%), que desciende hasta el 14.28\% entre 2013 y 2014. De manera similar sucede con "Araucano/s" e "Indio/s", cuya presencia decae con el paso del tiempo: pasan del $14.78 \%$ y $12.31 \%$ hasta el $2.04 \%$ y $0 \%$, respectivamente. Por el contrario, "Mapuche/s" cobra protagonismo en los últimos dos periodos analizados, subiendo del 22.17\% en 1950-1973; hasta el 69.56\% en 2010; y el 83.68\% en 2013-2014 (gráfico 1). 
Gráfico 1: Términos utilizados para denominar al colectivo mapuche.

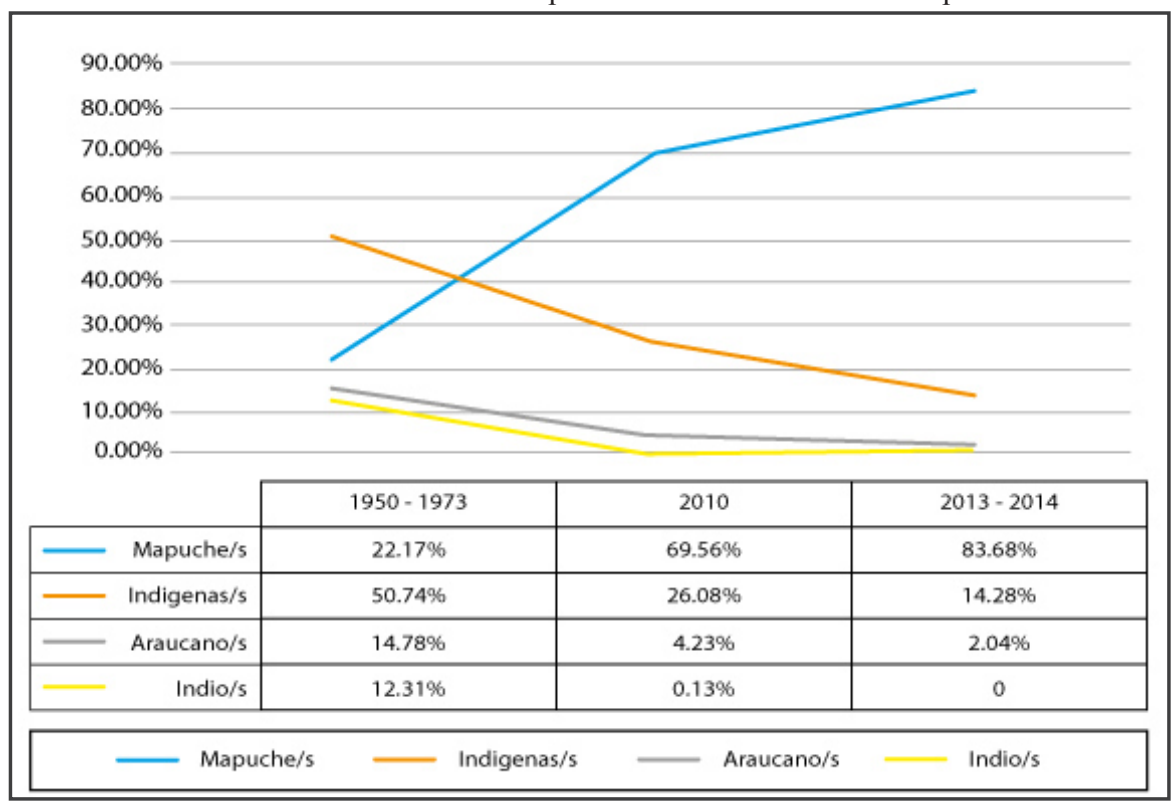

Fuente: Elaboración propia.

\subsection{Valoración, lenguaje y encuadre noticioso}

Al analizar el carácter evaluativo de las publicaciones, los datos reflejan que la valoración negativa hacia los mapuches crece con el paso de los años. De esta manera, se aprecia que entre 1950 y 1973 , el $15.80 \%$ de los textos analizados aporta una visión negativa, cifra que aumenta hasta el $40.47 \%$ en 2013 y 2014, periodo en el que predominan las informaciones sobre enfrentamientos, ataques incendiarios y acciones reivindicativas y protestas, de las que se les atribuye la responsabilidad a los indígenas. Por el contrario, el enfoque positivo disminuye ligeramente en estos mismos periodos, pasando del $23.90 \%$ al $14.89 \%$ (tabla 5).

Tabla 5: Carácter evaluativo de los textos periodísticos por periodos analizados.

\begin{tabular}{|c|c|c|c|}
\hline \multirow{2}{*}{ Valoración } & \multicolumn{3}{|c|}{ Periodo } \\
\cline { 2 - 4 } & $\mathbf{1 9 5 0 - 1 9 7 3}$ & $\mathbf{2 0 1 0}$ & $\mathbf{2 0 1 3 - 2 0 1 4}$ \\
\hline Neutral & $60.20 \%$ & $11.77 \%$ & $44.64 \%$ \\
\hline Positiva & $23.90 \%$ & $41.18 \%$ & $14.89 \%$ \\
\hline Negativa & $15.80 \%$ & $47.05 \%$ & $40.47 \%$ \\
\hline
\end{tabular}

Fuente: Elaboración propia.

Sin embargo, se ha detectado que la objetividad del lenguaje crece con el paso del tiempo, ya que se evitan las frases despectivas y se utilizan un mayor número de verbos en positivo. En 2013 y 2014, el $85.31 \%$ de los textos utiliza estructuras gramaticales simples, evita figuras retóricas y emplea una terminología más precisa, evitando interpretaciones erróneas. 
Al analizar el uso de los diferentes encuadres noticiosos, se ha observado que el frame que vincula el colectivo mapuche con el "Conflicto" es el que predomina sobre el resto de encuadres (16.39\%). Además, también tienen una alta presencia los encuadres que se refieren a "Procesamientos penales" (11.09\%); "Resolución de problemas y conflictos" (8.68\%); "Social" (8.03\%); "Judicatura" (6.91\%); "Atribución de responsabilidad" (5.94\%); "Ataques incendiarios" (5.30\%); "Cultura y Educación" (4.66\%); "Terrenos" (4.50\%); y "Acciones reivindicativas y protestas" (3.83\%) (gráfico 2). Cabe destacar que son muchos los textos que suman dos o más dimensiones temáticas. De hecho, se han contabilizado un total de 622 frames en las 340 publicaciones analizadas.

Gráfico 2: Puntuación media de cada encuadre noticioso.

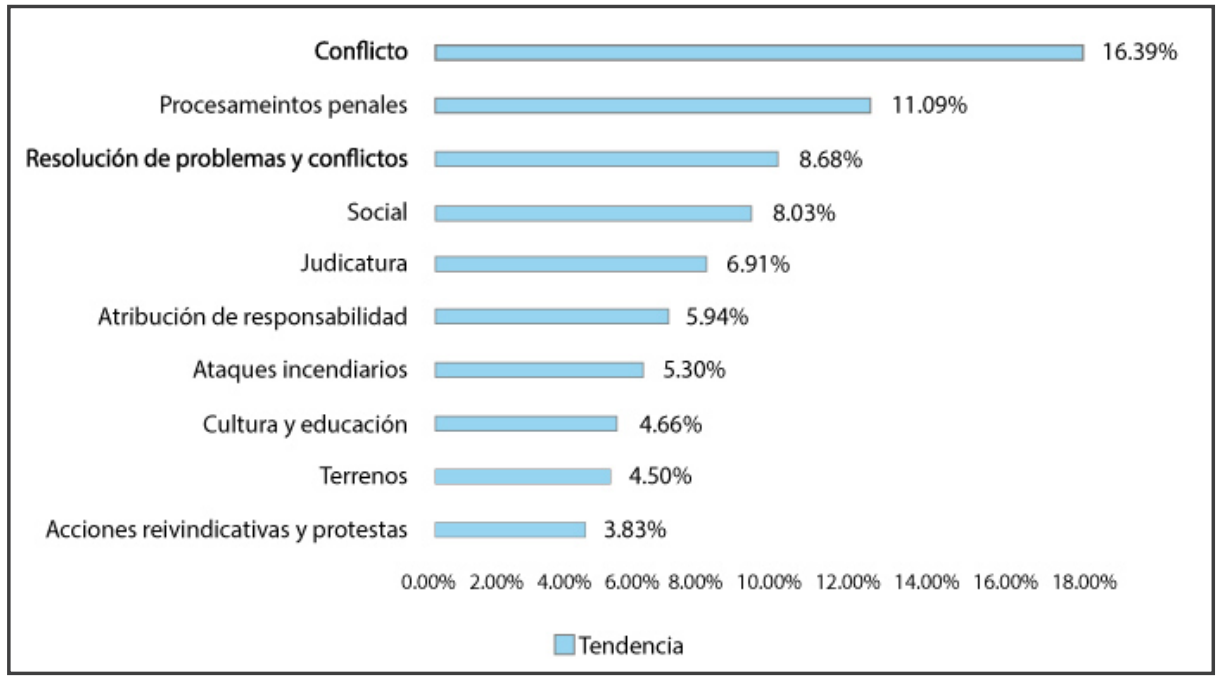

Fuente: Elaboración propia.

El frame "Conflicto" está presente en las tres etapas analizadas, aunque su frecuencia aumenta con el paso del tiempo. Entre 1950 y 1973, un $8.33 \%$ de publicaciones contiene este encuadre, que crece hasta el $27.27 \%$ en 2010 , para situarse en el $24.29 \%$ en 2013 2014. En esta última etapa, este frame comparte dimensión temática con otros textos que contienen atributos de valoración negativa, como los enfrentamientos y los ataques incendiarios, entre otros.

\section{Discusión y conclusiones}

La investigación desarrollada permite dar por conseguido el objetivo planteado inicialmente de analizar el tratamiento informativo de los mapuches en el diario El Mercurio durante los años 1950-1973, 2010, 2013 y 2014. Este estudio permite confirmar la primera hipótesis, ya que los textos publicados por este medio de comunicación sobre el colectivo indígena son de carácter despectivo y discriminatorio. La mayoría de los encuadres están vinculados con los conflictos (102 atribuciones), los procesamientos penales (69 atribuciones) 
y los ataques incendiarios (33 atribuciones), como ya se constató en un estudio piloto realizado en el marco del proyecto CONICYT PLU 140014, según el cual la mayoría de las menciones del pueblo mapuche en El Mercurio están vinculadas con algún aspecto de violencia, mientras que el segundo énfasis se concentra en los temas políticos, luego los económicos y finalmente los culturales. También están en línea con los resultados obtenidos por Foerster \& Vergara (2000), que advertían de la lucha de este colectivo por un mayor reconocimiento en la sociedad chilena.

En este sentido, el estudio corrobora la segunda hipótesis, ya que los conflictos, los procesamientos penales y los ataques incendiarios son los principales encuadres noticiosos que se derivan del tema mapuche en El Mercurio. Este diario ha popularizado la representación de un "conflicto mapuche" en las que las demandas políticas han sido anuladas, como si se tratase de problemas de seguridad pública suscitados entre activistas y propietarios agrícolas (Muñoz, 2010). No obstante, el tratamiento informativo de los araucanos se realiza a partir de una variedad de dimensiones temáticas y encuadres (hipótesis 3), que se han configurado con el paso del tiempo y que enfatizan la visión negativa del pueblo mapuche.

La manera cómo los medios de comunicación tratan las demandas y luchas por el reconocimiento cultural y político de los pueblos originarios es relevante "a causa de su impacto en la vida fuera de los medios" (Ferguson, 2007, p. 12). En el caso de El Mercurio, ha sido uno de los medios más críticos a las políticas de reconocimiento promovidas por los gobiernos democráticos, así como a las demandas políticas del movimiento mapuche (Foerster \& Vergara, 2000; Del Valle, 2005). De hecho, el sociólogo defensor de los derechos humanos de los pueblos indígenas, Rodolfo Stavengahen (2003), señaló que los medios de comunicación no habían prestado la necesaria atención a la situación de los derechos humanos de este colectivo, por lo que recomendaba que los medios ofreciesen una visión objetiva y equilibrada de los derechos indígenas.

Con estos datos es posible afirmar que la construcción de la agenda de El Mercurio en torno a este colectivo no es pluralista. El diario prioriza las connotaciones y los sucesos negativos para informar sobre los indígenas, a quienes atribuye disputas, peleas y confrontaciones. Este discurso se encuentra reforzado en algunas cartas al director y entrevistas a especialistas vinculados a la derecha. Este tratamiento informativo puede provocar diferentes efectos sociocognitivos en la sociedad, de acuerdo con las aportaciones de De Vreese (2003), McLeod \& Detenber (1999), Price, Nir \& Cappella, J. (2005) y Reese (2001), entre otros, los cuales demuestran que el uso de diferentes encuadres para dar cobertura a ciertos temas o grupos sociales influye en el procesamiento de la información y produce juicios y opiniones diferentes, dependiendo del encuadre utilizado.

En este sentido, el hecho de que los mapuches sean tratados, en general, con frames desfavorables y perjudiciales repercute en que las actitudes y creencias hacia este colectivo sea negativo por parte del público que consume este medio de comunicación. Debemos admitir que lo que está en juego no es sólo un problema de pobreza, de sectores económicos o socialmente desprotegidos que deben ser integrados en los mercados laborales, sino que 
se trata de una situación étnica que la sociedad chilena y su ordenamiento jurídico ha negado a lo largo del tiempo, de comunidades arrinconadas en su propio territorio en virtud de una política de Estado y de la acción de particulares que se beneficiaron de ella. De acuerdo con Aylwin (2000), debemos reconocer que existe de parte de la sociedad chilena y del Estado una deuda histórica con los mapuches que aún no ha sido saldada.

La cuestión estudiada no se agota en esta investigación sino que, muy al contrario, reafirma que se trata de un debate abierto y en evolución. En este proceso se hace patente la conveniencia de explorar nuevas vías de encuentro para redactar una "hoja de ruta" que permita buscar más puntos de convergencia entre el medio de comunicación que es objeto de estudio en la presente investigación y el pueblo mapuche. En esta "hoja de ruta" podríamos indicar, por ejemplo, qué acuerdos, diálogos o materias específicas permiten solucionar los problemas que hoy afectan a los mapuches y a su comunidad, entre otros puntos. De hecho, países como Nueva Zelanda y Canadá, entre otros, tienen una larga historia de resolución de conflictos a través del diálogo y la negociación (Aylwin, 2000).

\section{Fuentes consultadas}

Almonacid, F. (2009). El problema de la propiedad de la tierra en el sur de Chile (18501930). Historia, 1(42), 5-56.

Amolef, F. (2004). La alteridad en el discurso mediático. Los mapuches y la prensa chilena. Boletín IFP, 2(6). Recuperado de http://www.portalcomunicacion.com/dialeg/ paper/pdf/202_amolef.pdf

Andréu, J. (2002). Las técnicas de análisis de contenido. Una revisión actualizada. Granada: Fundación Centro de Estudios Andaluces.

Ardèvol-Abreu, A. (2015). Framing o teoría del encuadre en comunicación. Orígenes, desarrollo y panorama actual en España. Revista Latina de Comunicación Social, $70,423-450$.

Aruguete, N. (2017). Agenda setting y framing: un debate teórico inconcluso. Más poder local, 30, 36-42.

Asociación de Agencias de Medios, AAM (2018). Informe de la Asociación de Agencias de Medios. Recuperado de https://aam.cl/

Aylwin, J. (2000). Los conflictos en el territorio mapuche: antecedentes y perspectivas. Revista perspectivas, 3(2), 277-300.

Bardin, L. (2002). El análisis de contenido. Madrid: Akal. 
Bengoa, J. (2000). Historia del pueblo mapuche (siglo XIX y XX). Santiago de Chile: Editorial LOM.

Berelson, B. (1952). Content analysis in Communication Research. Illinois: Free Press.

Boccara, G. (1999). Etnogénesis mapuche: resistencia y restructuración entre los indígenas del centro-sur de Chile (siglos XVI-XVIII). The Hispanic American Historical Review, 79(3), 425-461.

Bryant, J. \& Miron, D. (2004). Theory and research in mass communication. Journal of Communication, 54(4), 662-704.

Carrasco, H. (2002). El discurso público mapuche: noción, tipos discursivos e hibridez. Estudios Filológicos, 37. Recuperado de https://scielo.conicyt.cl/scielo. php?script $=$ sci_arttext\&pid=S0071-17132002003700011

D'Angelo, P. (2002). News Framing as a Multiparadigmatic Research Program: A Response to Entman. International Communication Association, 52(4), 870-888.

De Vreese, C. (2003). Framing Europe. Television news and European integration. Ámsterdam: Aksant.

Del Valle, C. (2002). La estructura argumentativa de un tipo particular de discurso jurídico. El caso de cuatro demandas de reivindicación territorial mapuche (IX Región Chile). (En)clave Comahue. Revista Patagónica de Estudios Sociales, 7, 41-50.

Del Valle, C. (2003). La identidad en un doble juego: resistencia y apertura... o el aprendizaje del cinismo. La construcción social del llamado "conflicto mapuche" en Chile, en publicaciones electrónicas realizadas por los propios mapuches. Diálogos de la Comunicación, 66, 90-97.

Del Valle, C. (2004a). Genealogía crítica de la comunicación intercultural: mediocentrismo e invisibilización de lo étnico en los estudios interculturales. Sphera Pública. Revista de Ciencias Sociales y de la Comunicación, 4, 171-196.

Del Valle, C. (2004b). Los indígenas de Chile en las relaciones de sucesos españolas: Representación y memoria desde la interculturalidad. Zer. Revista de Estudios de Comunicación, 9(16), 1-9.

Del Valle, C. (2005). Interculturalidad e intraculturalidad en el discurso de la prensa: cobertura y tratamiento del discurso de las fuentes en el conflicto indígena mapuche, desde el discurso político. Redes.Com. Revista de Estudios para el Desarrollo Social de la Comunicación, 2, 83-111. 
Del Valle, C. (2007). Matriz Tetralémica, Cosmovisión y Salud/Enfermedad en la Cultura Mapuche (Chile): Complejidad, Ecología, Equidad/Diferencia y Comunicación. En F. Contreras (Dir.), Cultura Verde. Ecología, Medio Ambiente y Comunicación (pp. 141-153). Sevilla: Siranda Editorial, Publidisa.

Del Valle, C. (2015). La construcción del imaginario social de la justicia en los relatos periodísticos publicados por el diario El Mercurio de Chile, entre 1850 y 2014, en el contexto del conflicto Estado-nación y pueblo mapuche: Continuidades y cambios. Fondecyt n ${ }^{\circ} 1150666$.

Del Valle, C. (2016). Prensa, comunicación política y hegemonía: El rol de El Mercurio de Chile, como actor económico-político, en la construcción histórica del mapuche como bárbaro y terrorista. En C. Muñiz, A. Saldierna, F. Marañón \& L. Maldonado (Coords.), Estudios sobre comunicación politica en Latinoamérica (pp. 63-84). México, DF: Editorial Fontamara.

Del Valle, C. (2018). La producción del enemigo íntimo en la industria cultural chilena: Crítica a la certeza moral, la razón neoliberal y la sujeción criminal. En D. Caldevilla (Coord.), Perfiles actuales en la información y en los informadores (pp. 51-68). Madrid: Tecnos.

Del Valle, C. (2019). La criminalización radical del enemigo como estrategia del estado nacional y las élites en la lucha por las tierras indígenas. En A. Arévalo, G. Vilar \& M. García (Eds.), Comunicación y cambio social [En prensa]. Barcelona: Tirant lo Blanch.

Del Valle, C., Davinson, G. \& Maldonado, C. (2015). Discriminación y prejuicios culturales en sentencias penales a personas mapuche: Un análisis formal, racional y lógico al discurso jurídico-judicial en Chile. Revista Jurídicas, 12(1), 97-113.

Del Valle, C., Mellado, C., Salinas, P. \& González, G. (2011). La concentración de la propiedad de los medios de comunicación en Chile. De la propiedad al mercado de la publicidad: los desafíos pendientes en Sapiens. Revista Universitaria de Investigación, 12(1), 44-58.

Denegri, M., Chávez, D., Silva, F. \& Del Valle, C. (2015). Representaciones sociales en torno al "conflicto" Estado chileno-Pueblo Mapuche en jóvenes universitarios chilenos: un estudio con redes semánticas naturales. Investigación y Desarrollo, 23(2), 256-277.

Entman, R. (1993). Framing: toward a clarification of a fractured paradigm. Journal of Communication, 43, 51-68.

Ferguson, R. (2007). Los medios bajo sospecha. Ideología y poder en los medios de comunicación. Barcelona: Gedisa. 
Foerster G. \& Vergara, J. (2000). Los mapuches y la lucha por el reconocimiento en la sociedad chilena. XII Congreso Internacional: Derecho consuetudinario y pluralismo legal: desafios en el tercer milenio. Arica, Chile.

Ghanem, S. (2009). Filling in the Tapestry: The Second Level of Agenda Setting. En M. McCombs, D. Shaw \& D. Weaver (1997), Communication and Democracy. Exploring the intellectual frontiers in agenda setting theory (pp. 3-14). Nueva Jersey: Lawrence Erlbaum Associated.

Gitlin, T. (1980). The whole world is watching: Mass media in the making and unmaking of the new left. California: University of California Press.

Guo, L., Hong, T. \& McCombs, M. (2012). An Expanded Perspective on Agenda Setting Effects. Exploring the third level of agenda setting. Revista de Comunicación, 11, 51-68.

Hudson, E., Del Valle, C., Dussaillant, F. \& Browne, F. (2017-2018). Rutinas profesionales y pluralismo. Un estudio sobre las condiciones de producción informativa en la prensa nacional y en la prensa de la zona sur. Proyecto financiado por la Comisión Nacional de Investigación Científica y Tecnológica (CONICYT) PLU número 140014.

Iguarta, J. \& Humanes, M. (2004). Teoría e investigación en comunicación social. Madrid: Síntesis.

Koziner, N. (2013). Antecedente y fundamentos de la teoría del framing en comunicación. Austral Comunicación, 2(1). Recuperado de https://www.austral.edu.ar/ojs/index. php/australcomunicacion/article/view/53/49

Koziner, N. (2017). El tratamiento de las fuentes en la cobertura de la ley audiovisual argentina. El caso de la prensa económica (marzo-octubre 2009). Más Poder Local, 30, 43-51.

Koziner, N. \& Aruguete, N. (2016). El “7D” en los diarios económicos argentinos. Aplicación de encuadres noticiosos genéricos a la cobertura de la controversia judicial entre el Gobierno y el Grupo Clarín. Comunicación y Medios, 25(33), 7-34.

Krippendorff, K. (2002). Metodología del análisis de contenido. Teoría y práctica. Barcelona: Paidós.

Labrín, J. (2011). Análisis de la cobertura y tratamiento de conflictos indígenas (2010-2011). Santiago de Chile: Instituto de la Comunicación e Imagen. 
López-Rabadán, P. (2010). Nuevas vías para el estudio del framing periodístico: la noción de estrategia de encuadre. Estudios sobre el mensaje periodístico, 16, 235-258.

López, P. \& Vicente, M. (2008). Tendencias actuales en la investigación sobre framing: consolidación internacional y emergencia en la academia española. Investigar la comunicación. Congreso Internacional Fundacional de la Asociación Española de Investigación de la Comunicación. Santiago de Compostela, España.

Maher, M. (2001). Framing: An emerging paradigm or a phase of Agenda-Setting? En S. Reese, O. Gandy \& A. Grant (Eds.), Framing Public Life. Perspectives on media and our understanding of the social world (pp. 83-94). Nueva Jersey: Lawrence Erlbaum Associates.

Maldonado, C. (2014). El discurso hipertextual Mapuche como estrategia de resistencia y reivindicación cultural en el marco de las prácticas jurídico-judiciales referidas al conflicto Estado Nación y Pueblo Mapuche (tesis doctoral). Universidad Autónoma de Barcelona, España.

Maldonado, C.\& Del Valle, C. (2013). Medios de comunicación y narrativas hipertextuales. Lógicas del desplazamiento del "conflicto mapuche" al espacio virtual. Andamios, 10(22), 283-303.

Maldonado, C. \& Del Valle, C. (2016). Episteme decolonial en dos obras del pensamiento mapuche: re-escribiendo la interculturalidad. Chungara. Revista de Antropología Chilena, 48(2), 319-329.

Marín, J. \& Zamora, R. (2014). Aproximación integradora en la investigación sobre la teoría del framing desde su condición multiparadigmática. Disertaciones. Anuario Electrónico de Estudios en Comunicación Social, 7(1). Recuperado de https:// revistas.urosario.edu.co/index.php/disertaciones/article/view/3838

McCombs, M. (2006). Estableciendo la agenda. El impacto de los medios en la opinión pública y en el conocimiento. Barcelona: Paidós.

McCombs, M. \& Shaw, D. (1972). The Agenda-Setting function of the mass media. Public Opinion Quarterly, 36(2), Reino Unido: Oxford Journals.

McLeod, D. \& Detenber, B. (2006). Framing effects of television news coverage of social protest. Journal of Communication, 49(3), 3-23.

Muñiz, C. (2015). La política como debate temático o estratégico. Framing de la campaña electoral mexicana de 2012 en la prensa digital. Nueva Época, 23, 67-95. 
Muñiz, C., Igartua, J., Otero, J. \& Sánchez, C. (2008). El tratamiento informativo de la inmigración en los medios españoles. Un estudio comparativo de la prensa y televisión. Perspectivas de la Comunicación, 1(1), 97-112.

Muñoz, R. (2010). Discurso informativo y luchas por el reconocimiento el "conflicto mapuche" en El Mercurio y La Segunda (Chile, 2008-2009). Perspectivas de la Comunicación, 3(2), 29-47.

Oller, M. (2014). The triangle formed by framing, agenda-setting and metacoverage. Disertaciones. Anuario electrónico de estudios en Comunicación Social, 7(1). Recuperado de http://erevistas.saber.ula.ve/index.php/Disertaciones/

Price, V., Nir, L. \& Cappella, J. (2005). Framing public discussion of gay civil unions. The Public Opinion Quarterly, 69(2), 179-212.

Reese, S. (2001). Framing public life: A bridging model for media research (prólogo). En S. Reese, O. Gandy \& A. Grant (Eds.), Framing Public Life. Perspectives on media and our understanding of the social world (pp. 7-31). Nueva Jersey: Lawrence Erlbaum Associates.

Sádaba, M. (2001). Origen, aplicación y límites de la "teoría del encuadre" (framing). Comunicación y Sociedad, 24(2), 143-175.

Sáez, A. (1999). De la representació a la realitat. Barcelona: Dèria Editors.

Saiz, J., Merino, M. \& Quilaqueo, D. (2009). Meta-estereotipos sobre los indígenas mapuches en Chile. Interdisciplinaria. Centro Interamericano de Investigaciones Psicológicas y Ciencias Afines, 26(1), 23-48.

Saiz, J., Rapimán, M. \& Mladinic, A. (2008). Estereotipos sobre los Mapuches. Su reciente evolución. PSYKHE, 17(2), 27-40.

Salinas, C. \& Stange, H. (2009). Rutinas Periodísticas, Discusión y trayectos teóricos sobre el concepto y su estudio en la prensa chilena. Cuaderno de Trabajo. Santiago de Chile: Universidad de Chile.

Sánchez Aranda, J. (2005). Análisis de contenido cuantitativo de medios. En M. Berganza \& J. Ruiz (Coords.), Investigar en comunicación. Guía práctica de métodos y técnicas de investigación social en Comunicación (pp. 207-228). Madrid: McGraw-Hill.

Scheufele, D. (1999). Framing as a theory of media effects. Journal of Communication, 49(1), 103-122. 
Scheufele, D. (2004). Framing-effects approach: A theoretical and methodological critique. Communications. The European Journal of Communication Research, 29(4), 401-428.

Scheufele, D. (2009). Agenda-Setting, Priming, and Framing Revisited: Another Look at Cognitive Effects of Political Communication. Mass Communication \& Society, 3(2), 297-316.

Semetko, H. \& Valkenburg, P. (2000). Framing European politics: a content analysis of press and television news. Journal of Communication, 50(2), 93-109.

Stavengahen, R. (2003) Informe del Relator Especial sobre derechos humanos y las libertades fundamentales de los indigenas. Recuperado de http://www. politicaspublicas.net/panel/re/docs/222-informe-chile-re-stavenhagen-2003.html

Tankard, J. (2001). The empirical approach to the study of media framing. En S. Reese, O. Gandy \& A. Grant (Eds.), Framing Public Life. Perspectives on media and our understanding of the social world (pp. 95-106). Nueva Jersey: Lawrence Erlbaum Associates.

Thibodeau, P. (2017). The function of metaphor framing, deliberate or otherwise, in a social world. Metaphor and the Social World, 7(2), 270-290.

Toledo, V. (2005). Pueblo mapuche. Derechos colectivos y territorio: desafios para la sustentabilidad democrática. Santiago de Chile: Fundación Heinrich Böll, Fundación Ford. Recuperado de http://www.chilesustentable.net/wp-content/ uploads/2005/12/Pueblo-Mapuche-Derechos-Colectivos-y-Territorio-desafiospara-la-sustentabilidad-democratica-parte-1.pdf

Van Dijk, T. (2000). El estudio del discurso. En Van Dijk, T. (Comp.), El discurso como estructura y proceso (pp. 21-66). Barcelona: Gedisa.

Van Gorp, B. (2007). The Constructionist Approach to Framing: Bringing Culture Back. Journal of Communication, 57(1), 60-78.

Vicente, M. \& López, P. (2009). Resultados actuales de la investigación sobre framing: sólido avance internacional y arranque de la especialidad en España. Zer. Revista de Estudios de Comunicación, 14(26), 13-34.

Weaver, D. (2007). Thoughts on agenda setting, framing, and priming. Journal of Communication, 57(1), 142-147.

Weber, R. (1990). Basic content analysis. California: Sage. 